\section{Salud mental como componente de la salud universal*}

\section{Carissa F. Etienne ${ }^{1}$}

Forma de citar (artículo original) Etienne CF. Mental health as a component of universal health. Rev Panam Salud Publica. 2018;42:e140. https://doi.org/10.26633/RPSP.2018.140
La Organización Mundial de la Salud define a la salud como "un estado de completo bienestar físico, mental y social, y no solamente la ausencia de afecciones o enfermedades". La salud mental es, según esta definición, un componente fundamental de la salud. Una buena salud mental permite a las personas hacer frente al estrés normal de la vida, trabajar productivamente y realizar su potencial contribuyendo a sus comunidades.

En la Región de las Américas, los trastornos mentales, neurológicos y por uso de sustancias están entre los principales contribuyentes a la morbilidad, la discapacidad, las lesiones y la mortalidad prematura, y aumentan el riesgo de presentar otros problemas de salud; en 2013 entre 18,7\% y 24,2\% de la población de la Región ha tenido una enfermedad mental (1). Aunque la salud mental es un componente esencial de la salud, existen brechas críticas de tratamiento (es decir, personas con enfermedad mental grave que no reciben tratamiento) que llegan hasta el 73,5\% en los adultos y el $82,2 \%$ en los niños y adolescentes.

En el 2014, la Organización Panamericana de la Salud y sus Estados Miembros adoptaron el Plan de acción sobre salud mental para guiar las intervenciones de salud mental a realizar en la Región de las Américas entre el 2015 y el 2020 (2). El Plan identifica cuatro líneas estratégicas: 1) Formular e implementar políticas, planes y leyes en el campo de la salud mental para lograr una gobernanza apropiada y eficaz; 2) Mejorar la capacidad de respuesta de los sistemas y servicios de salud mental, a fin de proporcionar una atención integral y de calidad en el ámbito comunitario; 3) Elaborar e implementar programas de promoción y prevención en el ámbito de los sistemas y servicios de salud mental, con particular atención al ciclo de vida; y 4) Fortalecer los sistemas de información, la evidencia científica y las investigaciones.

La investigación es un componente básico para un adecuado proceso de elaboración de políticas, planificación y evaluación en materia de salud mental. La obtención de nuevos conocimientos a través de la investigación hace posible que toda política o decisión se base en datos contrastados y en prácticas óptimas. Por otro lado, la existencia de datos pertinentes y actualizados permite seguir de cerca las medidas aplicadas y determinar los servicios que pueden mejorarse. Actualmente la mayor parte de la investigación en salud mental discurre en países de altos ingresos y bajo el control de estos, un desequilibrio que es preciso corregir para que los países de ingresos bajos o medianos se doten de estrategias costo-efectivas y culturalmente apropiadas para atender a sus necesidades y prioridades en materia de salud mental.

El presente número sobre salud mental de la Revista Panamericana de Salud Pública pretende dar a conocer algunas de las iniciativas de investigación en la Región, en sus diversos contextos y culturas. Los artículos de este número están relacionados con la implementación del Plan de acción sobre salud mental e incluyen investigación culturalmente validada en relación con la carga de morbilidad y la evaluación de los progresos realizados en las políticas y la organización de servicios en materia de salud mental. Ocho de ellos son artículos de investigación original; algunos hacen un análisis exhaustivo sobre el impacto de la gobernanza en el desarrollo de los modelos de atención en Chile y Ecuador. Otros artículos documentan los avances y obstáculos de la reforma psiquiátrica en Brasil, así como la descentralización de servicios en Jamaica y El Salvador. Se describe además el interesante proceso de implementación de los hogares protegidos en Perú y el uso de la consultoría en atención primaria como una herramienta para disminuir la

\footnotetext{
Traducción oficial al español efectuada por la Organización Panamericana de la Salud. En caso de discrepancia prevalecerá la versión original (en inglés).

Directora, Organización Panamericana de la Salud, Oficina regional para las Américas de la Organización Mundial de la Salud, Washington, D.C., Estados Unidos de América.
} 
hospitalización en Chile. Por último, se revisa exhaustivamente la brecha de tratamiento en la Región, que tiene repercusiones en varios resultados, como el suicidio, fenómeno que es analizado en Ecuador y Colombia.

La excelente muestra de iniciativas de investigación que contiene este número muestra la enorme capacidad de la Región. Sin embargo, aún existen tareas pendientes. Es necesario mejorar la calidad y cantidad de los trabajos de investigación regional, fomentar una mayor cooperación entre instituciones, realizar investigaciones en diferentes contextos culturales y desarrollar métodos de caracterización de las disparidades en salud mental entre subpoblaciones en contextos de vulnerabilidad o poco representadas en los países.

Los artículos contenidos en este número muestran que, si bien la salud mental es un área relevante para la Región, aún existe un largo camino por recorrer para lograr que sea considerada como una prioridad por los gobiernos. Buscamos que en las Américas se valore, promueva y proteja la salud mental, se prevengan los trastornos mentales y por uso de sustancias, y que las personas con dichos trastornos puedan ejercer sus derechos humanos y accedan a una atención de salud y social oportuna y de alta calidad. Este número aporta evidencia científica que esperamos estimule a los actores regionales a buscar soluciones en sus contextos locales y a apoyar el desarrollo de la investigación.

\section{REFERENCIAS}

1. Organización Panamericana de la Salud. Salud en las Américas+, Edición 2017. Resumen: Panorama regional y perfiles de país. Washington, D.C.: OPS; 2017.

2. Organización Panamericana de la Salud. Plan de acción sobre salud mental 2015-2020. 53 Consejo Directivo, 66 Sesión del Comité Regional de la OMS para las Américas. Washington, DC: OPS; 2014. Disponible en: https://www.paho.org/hq/dmdocuments/2015/plan-ofaction-on-MH-2014.pdf. Acceso el 21 de agosto de 2018. 